\title{
ESTUDO DO CRESCIMENTO AUTOTRÓFICO DE TRÊS CEPAS DE MICROALGAS VISANDO A PRODUÇÃO DE BIODIESEL
}

\author{
V. F. de ALMEIDA, L. F. RIOS, R. M. FILHO, M. R. W. MACIEL \\ Universidade Estadual de Campinas, Faculdade de Engenharia Química \\ E-mail para contato: van.ferreira18@gmail.com
}

\begin{abstract}
RESUMO - Neste trabalho, estudou-se o crescimento de três diferentes cepas de microalgas: Chlorella vulgaris, Desmodesmus sp e Desmodesmus brasiliensis, visando a produção de biodiesel. As microalgas foram crescidas em cultivo autotrófico, no meio de cultivo BG-11. As taxas de crescimento destas microalgas foram calculadas e comparadas entre estas três cepas. Quanto maior for a taxa de crescimento da microalga, mais interessante é seu uso na produção de biodiesel, e mais viável se torna o processo de produção deste biocombustível. As microalgas que apresentaram maiores taxas de crescimento e menores tempo de duplicação foram Desmodesmus sp e Chlorella vulgaris.
\end{abstract}

\section{INTRODUÇÃO}

Com a diminuição das reservas de petróleo e a necessidade de encontrar fontes de energias competitivas e ambientalmente amigáveis, as energias renováveis vêm sendo altamente estudadas (Almeida et al., 2015). Dentre elas o biodiesel, um combustível biodegradável e não tóxico. Atualmente, há inúmeras opções de matéria prima para a produção de biodiesel. A microalga aparece como uma delas, e é bastante promissora, devido a sua alta atividade fotossintética, taxas de crescimento exponencial e alta produtividade de biomassa (Rios et al., 2015). O biodiesel produzido a partir de microalgas é classificado biocombustível de terceira geração, apresentando a vantagem de não competir com matérias primas que poderiam ser utilizadas para a alimentação (Galafassi et al., 2012). As microalgas acumulam lipídios em sua composição, que são extraídos e posteriormente esterificados, produzindo biodiesel (Francisco et al., 2014). Recentemente, a microalga Chlorella vulgaris vem sendo altamente estudada para a produção de biodiesel (Al-lwayzy et al., 2014; Farooq et al., 2015; Josephine et al., 2015), assim como a microalga Desmodesmus sp. (Rios et al., 2015; Ji et al., 2015). Porém, não se encontram dados na literatura sobre o cultivo da microalga Desmodesmus brasiliensis para a produção de biodiesel.

Neste trabalho, analisou-se o crescimento autotrófico destas três cepas de microalga: Chlorella vulgaris, Desmodesmus sp e Desmodesmus brasiliensis, comparando suas taxas de crescimento e visando a posterior produção de biodiesel. Um dos objetivos deste trabalho foi também avaliar o desempenho do crescimento da espécie Desmodesmus brasiliensis e comparar com as demais cepas de microalga, que já são conhecidas como sendo adequadas para a produção de biodiesel.

\section{MATERIAIS E MÉTODOS}




\subsection{Microalgas}

As microalgas Chlorella vulgaris e Desmodesmus brasiliensis foram doadas pelo grupo de Ecologia Aquática do Departamento de Ecologia e Biologia Evolutiva (DEBE) da Universidade Federal de São Carlos. Já a microalga Desmodesmus sp foi doada pelo Laboratório de Pesquisas com Organismos Aquáticos (LAPOA) do Grupo Integrado de Aquicultura e Estudos Ambientais (GIA) da Universidade Federal do Paraná (UFPR), Curitiba/Paraná.

\subsection{Meio de Cultivo}

As microalgas cresceram em um sistema de cultivo autotrófico, isto é, utilizando apenas a luz como fonte de energia para a fixação do $\mathrm{CO}_{2}$ e realizando fotossínteses para seu crescimento. Foi utilizado um sistema fechado para seu crescimento, ou seja, isoladas do meio externo e em recipientes transparentes. Utilizou-se o meio de cultivo líquido BG-11 (Rippka et al., 1979), preparado pela misturando todos os sais, metais necessários e ajustando o $\mathrm{pH}$ para um valor igual a 7,5. O meio apresenta a seguinte composição em mg/L: $\mathrm{NaNO}_{3}(1500$ $\mathrm{mg} / \mathrm{L}), \mathrm{K}_{2} \mathrm{HPO}_{4}(40 \mathrm{mg} / \mathrm{L}), \mathrm{CaCl}_{2} \cdot 2 \mathrm{H}_{2} \mathrm{O}(30 \mathrm{mg} / \mathrm{L}), \mathrm{Na}_{2} \mathrm{CO}_{3}(19 \mathrm{mg} / \mathrm{L}), \mathrm{MgSO}_{4} \cdot 7 \mathrm{H}_{2} \mathrm{O}(8$ $\mathrm{mg} / \mathrm{L}), \mathrm{C}_{6} \mathrm{H}_{8} \mathrm{O}_{7} \cdot \mathrm{H}_{2} \mathrm{O}(7 \mathrm{mg} / \mathrm{L})$, amônio, citrato férrico $(6 \mathrm{mg} / \mathrm{L}), \mathrm{H}_{3} \mathrm{BO}_{3} \quad(3 \mathrm{mg} / \mathrm{L})$, $\mathrm{MnCl}_{2} .4 \mathrm{H}_{2} \mathrm{O} \quad(2 \mathrm{mg} / \mathrm{L}), \quad \mathrm{Na}_{2}$ EDTA. $2 \mathrm{H}_{2} \mathrm{O} \quad(0.7 \mathrm{mg} / \mathrm{L}), \mathrm{Na}_{2} \mathrm{MoO}_{4} .2 \mathrm{H}_{2} \mathrm{O}(0.4 \mathrm{mg} / \mathrm{L})$, $\mathrm{ZnSO}_{4} .7 \mathrm{H}_{2} \mathrm{O} \quad(0.2 \mathrm{mg} / \mathrm{L}), \mathrm{CuSO}_{4} .5 \mathrm{H}_{2} \mathrm{O}(0.1 \mathrm{mg} / \mathrm{L})$ e $\mathrm{Co}\left(\mathrm{NO}_{3}\right)_{2} \cdot 6 \mathrm{H}_{2} \mathrm{O} \quad(0.05 \mathrm{mg} / \mathrm{L})$. Erlenmeyers, e demais elementos utilizados na preparação do meio de cultivo, e o próprio meio foram esterilizados em uma autoclave vertical (marca Phoenix Luferco, modelo AV-50 Plus) a uma temperatura de $121^{\circ} \mathrm{C}$ durante 15 minutos.

\subsection{Reprodução das Microalgas}

Cada cepa de microalga foi reproduzida no meio de cultivo BG-11 em Erlenmeyers de $250 \mathrm{~mL}$ de capacidade e em duplicata, totalizando seis experimentos. Para garantir uma fonte de luz constante $\left(72 \mu \mathrm{Em}^{-2}\right)$ estas foram mantidas em uma câmara com um banco de luzes com luz por 24 horas.

\subsection{Crescimento das Microalgas}

O crescimento das microalgas Chlorella vulgaris, Desmodesmus sp e Desmodesmus brasiliensis foi monitorado diariamente durante 14 dias através de um espectofotômetro UV/visível (marca Agilent Technologies, modelo Cary 60). As leituras foram feitas em comprimento de 682, 684 e $680 \mathrm{~nm}$, para as espécies Chlorella vulgaris, Desmodesmus sp e Desmodesmus brasiliensis, respectivamente. Para determinar a densidade celular em $\mathrm{mg} / \mathrm{mL}$, uma curva de calibração foi construída.

\subsection{Recuperação da Biomassa}

Quando as microalgas atingiram 14 dias de crescimento, foram retiradas do meio de cultivo com a finalidade de evitar morte celular. Para retirar recuperar a biomassa proveniente das microalgas, foi utilizada uma centrífuga (marca eppendorf, modelo 5810R). O processo de centrifugação foi realizado a temperatura ambiente, a uma velocidade de rotação de 5000 
rpm, por 30 minutos. Após a centrifugação, obtêm-se duas fases: a fase superior, composta pelo meio de cultivo, e a fase inferior que contém a biomassa. Em seguida, a biomassa foi separada e secada em uma estufa a temperatura de $104{ }^{\circ} \mathrm{C}$, durante $24 \mathrm{~h}$, até a obtenção de um peso constante.

\subsection{Determinação da Taxa de Crescimento das Microalgas}

A taxa de crescimento das microalgas foi determinada pela da linearização da curva de crescimento, plotando esta curva em um gráfico semilogarítmico. A taxa de crescimento foi calculada através da Equação 1.

$$
\frac{d X}{d t}=\mu
$$

$\mathrm{Na}$ qual X é a concentração da célula $(\mathrm{mg} / \mathrm{mL})$, t é o tempo e $\mu$ é a taxa de crescimento específica. A definição de $\mu$ é dada na Equação 2 a seguir:

$$
\mu=\frac{\ln \frac{X_{t}}{X_{t 0}}}{t-t_{0}}
$$

Do gráfico semilogarítmico, pode-se obter a taxa de crescimento das microalgas pela inclinação da curva na fase de crescimento exponencial, como mostrado na Equação 3.

$$
X=X_{0}+\mu_{t}
$$

\subsection{Tempo de Duplicação das Microalgas}

O tempo de duplicação das microalgas $\left(\mathrm{t}_{\mathrm{d}}\right)$ é o tempo gasto para a divisão de uma célula da microalga. $O$ cálculo de $t_{d}$ é apresentado na Equação 4:

$$
t_{d}=\frac{\ln 2}{\mu}
$$

\section{RESULTADOS E DISCUSSÃO}

A Figura 1 abaixo apresenta as curvas de crescimento das microalgas. Em cada um dos gráficos a, b e c se encontram duas curvas, sendo que cada uma representa o crescimento da mesma cepa, crescidas em duplicata ( 1 e 2) em Erlenmeyer diferentes. Todas as curvas apresentam a forma característica de uma "curva S", composta por quatro fases bem definidas: fase de latência, na qual as células se adaptam ao meio; uma fase de crescimento exponencial, na qual as células se multiplicam em sua taxa máxima; uma fase estacionária, na qual as células crescem em uma taxa igual a sua taxa de morte; e a fase de morte, na qual a taxa de crescimento de células decresce (não mostrado na Figura 1).

Figura 1 - Curva de crescimento das microalgas: a) Chlorella vulgaris, b) Desmodesmus sp e c) Desmodesmus brasiliensis. 

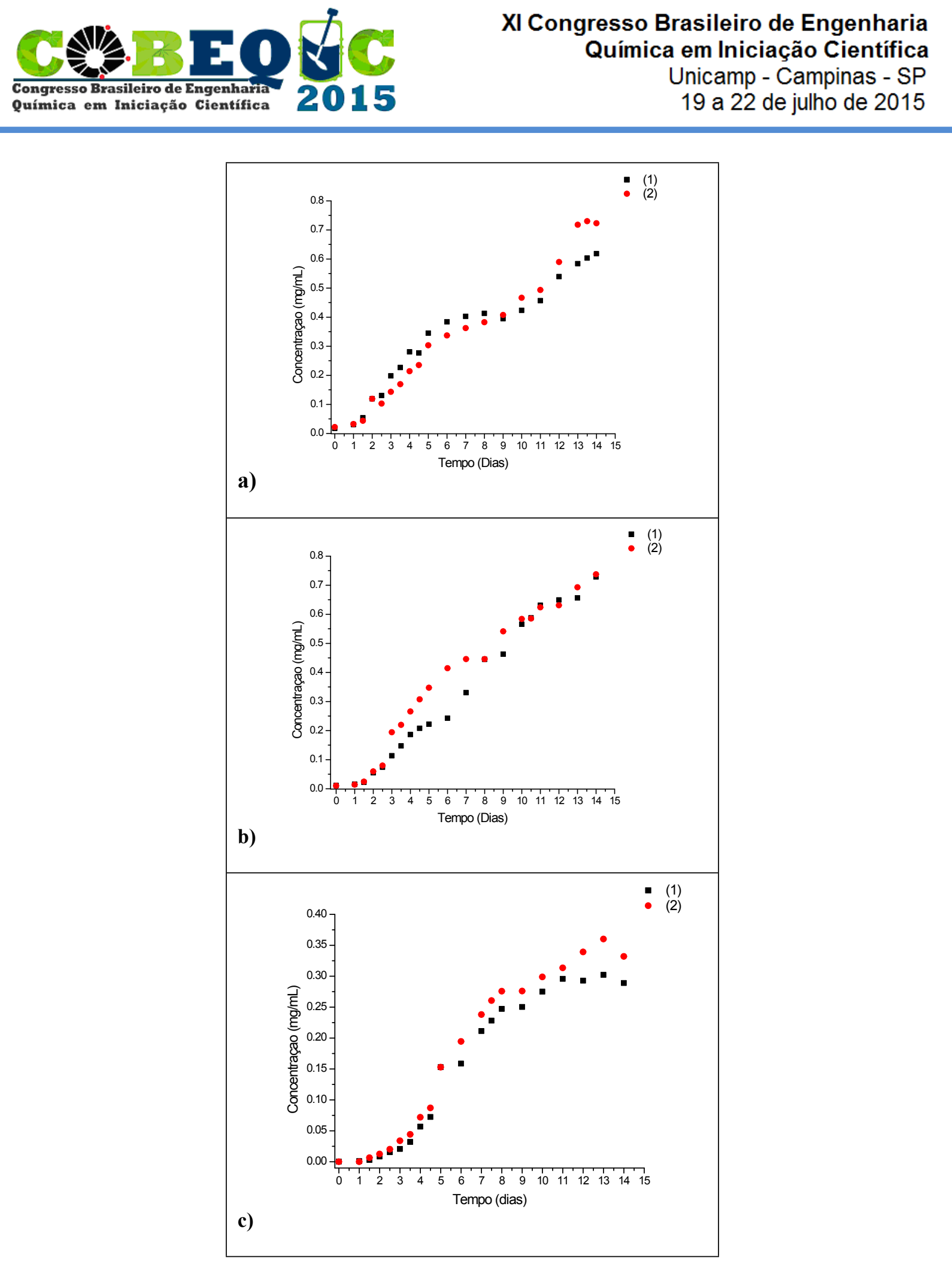

As velocidades máximas de crescimento se encontram na fase exponencial. Para a Desmodesmus sp. e Chorella vulgaris estas se encontram entre os dias 2 a 5 do cultivo. Já para a Desmodesmus brasilienses entre os dias 4 e 7 de cultivo. Comparando a fase de crescimento exponencial, é possível observar que as microalgas das espécies Chlorella vulgaris e Desmodesmus sp. cresceram mais rápido que a microalga Desmodesmus brasiliensis. Para exemplificar, pode-se ver que no tempo de crescimento igual a 5 as espécies com taxa de crescimento mais rápida já haviam atingido a concentração que variava de $0,20 \mathrm{a}$ $0,35 \mathrm{mg} / \mathrm{mL}$, enquanto a microalga Desmodesmus brasiliensis só tinha atingido uma concentração de $0,15 \mathrm{mg} / \mathrm{mL}$. No final do cultivo, as microalgas Chlorella vulgaris e 
Desmodesmus sp. atingiram uma concentração máxima entre 0,60 a $0,75 \mathrm{mg} / \mathrm{mL}$, enquanto a microalga Desmodesmus brasilienses chegou apenas em aproximadamente $0,30 \mathrm{mg} / \mathrm{mL}$. Com isto, pode-se afirmar que as microalgas Chlorella vulgaris e Desmodesmus sp atingiram maiores crescimentos celulares, quando cultivadas no meio BG-11. O valor da concentração de biomassa dessas algas foi aproximadamente o dobro em concentração do que a cepa Desmodesmus brasilienses. A seguir, na Tabela 1 são mostradas as taxas de crescimento máximas e o tempo de duplicação das três cepas de microalgas.

Tabela 1 - Taxa de crescimento máximo e tempo de duplicação das microalgas.

\begin{tabular}{|c|c|c|c|c|}
\hline \multirow{2}{*}{ Dados } & Cultivo & Chlorella vulgaris & Desmodesmus sp & $\begin{array}{c}\text { Desmodesmus } \\
\text { brasiliensis }\end{array}$ \\
\hline \multirow{3}{*}{$\boldsymbol{\mu}_{\max }\left(\mathbf{d i a}^{-1}\right)$} & 1 & 0,076 & 0,089 & 0,061 \\
\cline { 2 - 5 } & 2 & 0,103 & 0,113 & 0,078 \\
\cline { 2 - 5 } & Média & 0,089 & 0,101 & 0,070 \\
\hline \multirow{3}{*}{$t_{\mathbf{d}}(\mathbf{h})$} & 1 & 219,76 & 187,13 & 272,71 \\
\cline { 2 - 5 } & 2 & 161,51 & 147,22 & 213,28 \\
\cline { 2 - 5 } & Média & 190,63 & 167,17 & 242,30 \\
\hline
\end{tabular}

As cepas de microalgas foram crescidas em duplicata no mesmo meio de cultivo (BG11), sendo cada cepa de uma mesma espécie identificada pelos números " 1 " e "2". A partir da Tabela 1, pode-se observar que todas as cepas de microalgas numeradas como "1" obtiveram menores taxa de crescimento e maiores tempo de duplicação, comparando-se com microalgas da mesma espécie numeradas como " 2 ". Calculando-se a média dos valores obtidos para uma mesma espécie, a microalga Desmodesmus brasilienses apresentou taxas de crescimento e tempo de duplicação menos favoráveis dentre as três cepas. Já a microalga Desmodesmus sp. apresentou os valores mais favoráveis de taxas de crescimento e tempo de duplicação, seguida da microalga Chlorella vulgaris.

\section{CONCLUSÃO}

Na média, as microalgas com maior taxa de crescimento e menor tempo de divisão são as cepas de Desmodesmus sp. e Chlorella vulgaris, sendo que a microalga Desmodesmus sp., mostrou melhor crescimento no meio BG-11. A microalga Desmodesmus brasiliensis, obteve a menor taxa de crescimento e o maior tempo de divisão celular, isto quer dizer que esta cepa não tem um bom desempenho no meio BG-11. Com base nestes resultados obtidos, foi possível concluir que as espécies de microalga mais adequadas no crescimento em meio de cultivo BG-11visando a produção de biodiesel são a Desmodesmus sp. e Chlorella vulgaris. Contudo, também se deve levar em consideração a porcentagem de lipídios contidos nas microalgas, visto que o lipídio é a matéria prima deste biocombustível. Portanto, a microalga Desmodesmus brasiliensis ainda não deve ser descartada para a sua utilização na produção de biodiesel. Além disso, também seria possível fazer o estudo do crescimento de esta cepa em meio mixotrófico, isto é, com fonte de luz e de carbono, para analisar se suas taxas de crescimento e tempo de duplicação apresentariam melhores resultados.

\section{AGRADECIMENTOS}


Os autores agradecem o financiamento da Fundação de Amparo à Pesquisa do Estado de São Paulo (FAPESP), Processo número 2014/10064-9.

\section{REFERENCIAS}

AL-LWAYZY, S. H.; YUSAF, T.; AL-JUBOORI, R. A. Biofuels from the fresh water microalgae Chlorella vulgaris (FWM-CV) for diesel engines. Energies, v. 7, p. 1829-1851, 2014.

ALMEIDA, V. F. de.; GARCÍA-MORENO, P. J.; GUADIX, A.; GUADIX, E. M. Biodiesel production from mixtures of waste fish oil, palm oil and waste frying oil: Optimization of fuel properties. Fuel Processing Technology, v. 133, p. 152-160, 2015.

FAROOQ, W.; MOON, M.; RYU, B. G.; SUH, W. I.; SHRIVASTAV, A.; PARK, M. S.; MISHRA, S. K.; YANG, J. W. Effect of harvesting methods on the reusability of water for cultivation of Chlorella vulgaris, its lipid productivity and biodiesel quality. Algal Research, v. 8, p. 1-7, 2015.

FRANCISCO, É. C.; FRANCO, T. T.; MARONEZE, M. M.; ZEPKA, L. Q.; JACOBLOPES, E. Produção de biodiesel de terceira geração a partir de microalgas. Ciência Rural, v. 45, p. 349-355, 2015.

GALAFASSI, S.; CUCCHETTI, D.; PIZZA, F.; FRANZOSI, G.; BIANCHI, D.; COMPAGNO, C. Lipid production for second generation biodiesel by the oleaginous yeast Rhodotorula graminis. Bioresource technology, v. 111, p. 398-403, 2012.

JI, F.; ZHOU, Y.; PANG, A.; NING, L.; RODGERS, K.; LIU, Y.; DONG, R. Fed-batch cultivation of Desmodesmus sp. in anaerobic digestion wastewater for improved nutrient removal and biodiesel production. Bioresource technology, v. 184, p.116-122, 2015.

JOSEPHINE, A.; NIVEDITHA, C.; RADHIKA, A.; SHALI, A. B.; KUMAR, T. S.; DHARANI, G.; KIRUBAGARAN, R. Analytical evaluation of different carbon sources and growth stimulators on the biomass and lipid production of Chlorella vulgaris-Implications for biofuels. Biomass and Bioenergy, v. 75, p. 170-179, 2015.

RIOS, L. F.; KLEIN, B. C.; LUZ, L. F.JR.; MACIEL, M. R. W.; FILHO, R. M. Influence of Culture Medium on Desmodesmus sp. Growth and Lipid Accumulation for Biodiesel. Production Chemical Engineering Transactions, v. 43, 2015.

RIPPKA, R.; DERUELLES, J.; WATERBURY, J.B.; HERDMAN, M.; STANIER, R. Y. Generic assignments, strain histories and properties of pure cultures of cyanobacteria. Journal of General Microbiology, v. 111, p. 1-61, 1979. 